\title{
Psychometric evaluation of the Iranian version of brief fear of negative evaluation scale-straightforward item (BFNE-S): A validation study
}

\author{
Azadeh Tavoli ${ }^{1}$, Ali Montazeri*2 (D) \\ Received: 15 Jun 2019 \\ Published: 4 Apr 2020
}

\section{Abstract}

Background: The Brief Fear of Negative Evaluation Scale-Straightforward Item (BFNE-S) is an internationally recognized tool for measuring fear of negative evaluation. The current investigation was designed to assess the reliability and validity of the Persian version of BFNE-S in a non-clinical sample in Iran.

Methods: A cross-sectional study was conducted on a sample of university students. They completed the Iranian version of the BFNE-S and the Social Phobia Inventory (SPIN). Internal consistency was assessed using the Cronbach's alpha coefficient. Validity was examined using known groups comparison to test how well the questionnaire differentiates between subgroups of the study sample that differed in gender. In addition convergent validity was performed to examine the correlation between the BFNE-S and scores derived from the SPIN. Furthermore, the structural validity of the questionnaire was examined by performing confirmatory factor analysis using the LISREL 8.8 software.

Results: A total of 150 university students participated in the study. The results obtained from reliability analysis indicated that the Iranian version of the BFNE-S had desirable internal consistency. The Cronbach's alpha coefficient was 0.89. Satisfactory discriminant and convergent validity of the questionnaire also were established. The BFNE-S score was significantly higher in female respondents compared to male respondents as hypothesized $(\mathrm{P}=0.008)$. The BFNE-S was correlated to the Iranian version of the Social Phobia Inventory (SPIN) in the expected direction $(\mathrm{r}=0.58, \mathrm{P}<0.001)$. The confirmatory factor analysis indicated a good fit to data lending support to its original one-dimensional structure.

Conclusion: The findings indicated that the Iranian version of the BFNE-S was a valid measure of fear of negative evaluation. Since the scale showed a unitary factor structure, the theoretical basis for the BFNE-S is confirmed.

Keywords: Psychometrics, Surveys and questionnaires, Factor analysis, Fear, Iran

Conflicts of Interest: None declared

Funding: None

*This work has been published under CC BY-NC-SA 1.0 license.

Copyright $\odot$ Iran University of Medical Sciences

Cite this article as: Tavoli A, Montazeri A. Psychometric evaluation of the Iranian version of brief fear of negative evaluation scale-straightforward item (BFNE-S): A validation study. Med J Islam Repub Iran. 2020 (4 Apr);34:29. https://doi.org/10.47176/mjiri.34.29

\section{Introduction}

Social anxiety disorder (SAD), formerly termed social phobia a persistent and overwhelming fear of being embarrassed or humiliated in social situations, in conjunction

Corresponding author: Dr Ali Montazeri, montazeri@acecr.ac.ir

1. Department of Psychology, Faculty of Educational Science and Psychology, Alzahra University, Tehran, Iran

2. Population Health Research Group, Health Metrics Research Center, Iranian Institute for Health Sciences Research, ACECR, Tehran, Iran with avoidance of (or distress in) situations in which this fear is activated (1). During social interaction, people with social phobia may interpret a vague situation as indicative

$\uparrow$ What is "already known" in this topic:

The Brief Fear of Negative Evaluation Scale (BFNE) is a wellknown instrument for measuring social anxiety and was validated and used in many countries, including Iran. However, several different versions including the 'Straightforward Items' of the instrument exist. It does not include reverse-worded items and has strong relationships with theoretically related measures.

$\rightarrow$ What this article adds:

This study validated the Brief Fear of Negative Evaluation Scale-Straightforward Items (BFNE-S) in Iran and now can be used to measure social anxiety. 
of negative happening. Furthermore, social phobia might cause mildly negative social events to be interpreted in a negative self-referential and catastrophic way. As such, it is argued that fear of negative evaluation (FNE) is a central characteristic of social anxiety $(2,3)$. In fact, fear of negative evaluation is a cognitive process and refers to the irrational beliefs related to being judged disparagingly or hostilely by others (4).

There are a number of scales that assess fear of negative evaluation including the Fear of Negative Evaluation Scale (FNE) (5) and the Brief version of the Fear of Negative Evaluation Scale (6), the Brief version of the Fear of Negative Evaluation Scale-Straightforward Items-BFNE-S (7), and the brief version of the Fear of Negative Evaluation Scale-revised (BFNE-II) (8).

The psychometric properties of the brief version of the Fear of Negative Evaluation scale are well documented (4, 9-11). For instance, the study of the Iranian version of BFNE proved that it is a reliable and valid instrument for measuring social anxiety and its structural validity supported a two-factor solution for the questionnaire (11). Similarly, Rodebaugh et al. (7) and Duck et al. (12) found a two-factor solution for the scale indicating that positive and reverse-worded items best fit the data. However, it was suggested that since a two-factor structure of the questionnaire does not confirm to the prior theoretically based assumptions of the BFNE unity $(6,13)$, one should change negative worded items to straightforward items similar to other items within the questionnaire or remove these negative items. Thus, some investigators such as Rodebaugh et al. (7), and Weeks et al. (4) recommended that reverse-worded items should be deleted from the questionnaire and its scoring. This version of the questionnaire is known as the BFNE-S. Also there is another form of the BFNE measure that in which reverse-worded items are reworded. This revised version of the BFNE is known as the BFNE-II (8). A chronological perspective of the instrument development is presented in Table 1. However, recently even those who suggested changing negative worded items to straightforward items provided more definitive recommendations about these alternatives and showed that the 12-item version of the BFNE was inferior or comparable to the 8-item version (14). Thus, it is possible to summarize that the current evidence support the advantage of the 8-item straightforwardly worded BFNE version. Indeed present study aimed to examine psychometric properties of the BFNE-S in Iran. This might help practitioners and researcher to be able to measure social anxiety disorder in practice and research settings. As indicated previously we translated the BFNE into Persian language and reported its psychometric properties in a sample of Iranian population elsewhere (11).

\section{Methods}

Participants and data collection: The Iranian version of the Brief version of the Fear of Negative Evaluation Scale-Straightforward items (BFNE-S) was administered to a sample of university students in Tehran, Iran. The data was colleted during Spring 2015. There were no restrictions in recruiting the study sample with regard to age, gender and study discipline. All students were asked to answer a brief demographic questionnaire, the Social Phobia Inventory (SPIN), and the BFNE-S.

\section{Instruments}

The Social Phobia Inventory (SPIN): The SPIN is selfreported scale that assesses fear avoidance and physiological features of social phobia, measures illness severity, and is sensitive to change over time (15). The SPIN contains 17 items. Each item is rated on a 5-point Likert scale ranging from 0 to 4 ; giving a total score ranging from 0 to 68 (15). Results of a validation study indicated that the SPIN demonstrated acceptable psychometric properties and showed that it is a suitable scale for screening and evaluating social anxiety severity in an Iranian population (16).

The Brief version of the Fear of Negative Evaluation Scale- Straightforward items (BFNE-S): The BFNE-S (7) is a revised version of the Brief Fear of Negative Evaluation scale (6). The Persian version of the questionnaire was provided from our previous work (11). In fact we selected the relevant items form the Persian version of the BFNE to provide the BFNE-S. It contains 8 items, and each item is rated on a 5-point Likert scale ranging from 0 (not at all) to 4 (extremely) (7). The scores on this questionnaire range from 0 to 32 with higher scores indicating worse conditions.

\section{Statistical analysis}

A number of statistical tests were used to explore the data. To analyze the data SPSS (version 13) was used to explore the data, estimate internal consistency, and perform known groups comparison. The confirmatory factor analysis was performed using LISREL 8.8 software.

1. Internal consistency was assessed by estimating the Cronbach's alpha coefficient. The value of 0.7 or above was considered satisfactory (17).

2. In order to assess validity the following tests were performed:

(i) Known-groups comparison was performed to examine how well the questionnaire discriminates between subsamples of the study participants who differed in gender. Studies demonstrated that there are meaningful differences between men and women in the severity of social phobia in non-clinical populations $(8,18,19)$. Thus it was hypothesized that women would score higher than men on

Table 1. Chronology of the instrument development

\begin{tabular}{lccc}
\hline Scale & Author (year) & Number of items & Content \\
\hline FNE & Watson and Friend (1969) & 30 & Straightforward and reverse-worded items \\
BFNE & Leary (1983) & 12 & Straightforward and reverse-worded items \\
BFNE-S & Rodebaugh et al. (2004) & 8 & Straightforward items \\
BFNE-II & Carleton, Collimore, \& Asmundson (2007) & 12 & Straightforward items \\
\hline
\end{tabular}


the BFNE.

(ii) The correlation between the BFNE-S and the SPIN was examined to establish convergent validity. It was expected that the BFNE-S would positively correlate with the SPIN. The Pearson correlation coefficient of 0.40 or above was thought acceptable (20).

3 . Furthermore, the structural validity of the questionnaire was examined by performing the confirmatory factor analysis in order to confirm the original structure of the instrument and goodness of fit indexes were reported (21).

\section{Ethics}

The ethics committee of the Payam Noor University approved the study. All participants were informed about the aims and methods of the study. Also verbal consents obtained from all participants prior to interviews. The ethics committee approved to obtain the consents verbally in advance.

\section{Results}

A toal of 150 students were studied. The mean age of the respondents was $23.3(\mathrm{SD}=2.9)$ years, $75.3 \%$ were female, and mostly single $(72.7 \%)$. The characteristics of the study sample are presented in Table 2.

The internal consistency of the BFNE-S as estimated by the Cronbach's alpha coefficient, showed a good result $($ Cronbach's alpha coefficient $=0.89$ ).

Validity of the BFNE-S was examined using the known groups comparison and convergent validity. The BFNE-S discriminated well between subgroups of the study sample that differed in gender indicating that the BFNE-S score was higher in women as expected. The results are shown in Table 3.

The correlation between the BFNE-S and the Iranian version of Social Phobia Inventory (SPIN) was calculated in order to examine convergent validity and as expected the BFNE-S well correlated (strong) with the SPIN (Pearson correlation coefficient $=0.58, \mathrm{P}<0.001$ ).

Finally we performed the confirmatory factor analysis
Table 2. Demographic characteristics of the study sample $(n=150)$

\begin{tabular}{lccc}
\hline & & Number & $\%$ \\
\hline Age & Mean (SD) & $23.3(2.9)$ & - \\
Gender & Range & $19-36$ & - \\
& Male & 37 & 24.7 \\
Marital status & Female & 113 & 75.3 \\
& Single & 109 & 72.7 \\
Education & Married & 41 & 27.3 \\
& First year & 17 & 11.3 \\
& Second year & 40 & 26.6 \\
& Third year & 59 & 39.3 \\
& Forth year & 34 & 22.7 \\
\hline
\end{tabular}

and the results showed acceptable goodness of fit indexes for the data confirming the original one-dimensional structure of the instrument. The findings are shown in Table 4. Also the output for the analysis is presented in Figure 1.

\section{Discussion}

The current investigation was designed to examine the reliability and validity of the Persian version of the BFNE$\mathrm{S}$ in a non-clinical sample in Iran. The BFNE-S is a revised version of the Brief Fear of Negative Evaluation (6). In general, the findings showed promising results and were comparable with most studies on the topic among other populations $(14,22,23)$. Also the results showed that the BFNE-S includes one factor as indicated in previous investigations $(14,18,19)$. The Iranian version of the BFNE-S proved to be acceptable to students and as indicated by other investigators, its reliability and internal consistency was found to be satisfactory.

The known groups comparison indicated that the Iranian version of the BFNE-S is a valid instrument for measuring fear of negative evaluation. It was able to discriminate well between male and female respondents. This is in line with recent evidence where significant difference in fear of negative evaluation was observed between men and women $(8,18,19)$. Carleton points out that usually women show significantly higher scores in fear of negative evaluation (8). Similarly studies showed that usually girls report significantly more social anxiety than boys, espe-

Table 3. Comparison of the BFNE-S, and the SPIN among males and females

\begin{tabular}{|c|c|c|c|}
\hline & $\begin{array}{c}\text { Male } \\
(n=37)\end{array}$ & $\begin{array}{l}\text { Female } \\
(n=113)\end{array}$ & $\mathrm{P}$ \\
\hline \multicolumn{4}{|c|}{ Brief version of the Fear of Negative Evaluation Scale- straightforward items (BFNE-S) } \\
\hline Mean (SD) & 6.2 & 9.5 & 0.008 \\
\hline Range & $0-19$ & $0-25$ & \\
\hline \multicolumn{4}{|c|}{ Social Phobia Inventory (SPIN) } \\
\hline Mean (SD) & $7.03(6.4)$ & $10.05(8.5)$ & 0.049 \\
\hline Range & $0-27$ & $0-39$ & \\
\hline
\end{tabular}

Table 4. Fit indices and their acceptable thresholds obtained from in confirmatory factor analysis for the Iranian version of BFNE confirming the original conceptual model of the instrument $\mathrm{S}$

\begin{tabular}{lcc}
\hline Fit Index & Results & Acceptable range* \\
\hline Model $\chi^{2}$ & $31.7(\mathrm{P}=0.016)$ & $>0.05$ \\
Relative $\chi^{2}\left(\chi^{2} / \mathrm{df}\right)$ & 2.87 & $2.0-5.0$ \\
RMSEA (Root Mean Square Error of Approximation) & 0.076 & Good: $0.08>$, medium: $0.1-0.08$ and poor: $0.1<$ \\
GFI (Goodness-of-fit index) & 0.95 & $>0.8$ \\
SRMR (Standardized root mean square residual) & 0.038 & $<0.05$ \\
NFI (Normed fit index) & 0.97 & $>0.5$ \\
NNFI (Non-normed fit index) & 0.98 & $>0.5$ \\
CFI (Comparative fit index) & 0.98 & $\geq 0.9$ \\
\hline
\end{tabular}

* Reference (21). 


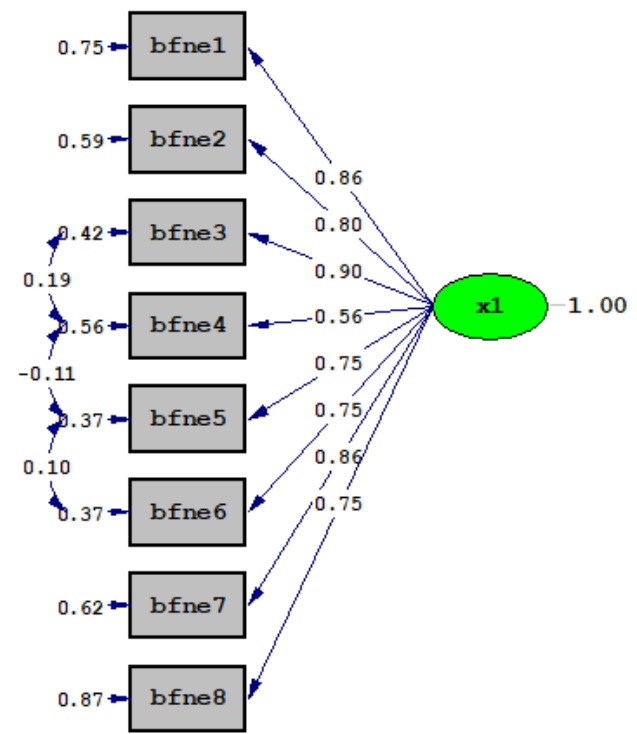

Chi-Square $=31.74, \mathrm{df}=17, \mathrm{P}$-value $=0.01622, \mathrm{RMSEA}=0.076$

Fig. 1. The output of confirmatory factor analysis for the Iranian version of BFNE-S confirming the original structure of the instrument

cially with regard to fear of negative evaluation $(24,25)$.

The BFNE-S showed a positive and significant correlation with the SPIN. In fact this indicates that those who were more socially anxious showed higher levels of fear of negative evaluation. Thus, this could be regarded as further proof to suggest that the BFNE-S is a valid instrument for measuring fear of negative evaluation. This finding is consistent with previous investigations where it was demonstrated that a positive relationship between the BFNE and the SPIN exist $(6,8,11,18,19,26)$.

Currently the lifetime prevalence of social anxiety disorder is $10.1 \%$ in Iran. Social fears considerably vary in different cultures .A social behavior is considered as normal in a culture, while the same behavior in another culture is considered to be abnormal or extreme (27). Persian and Iranian culture has been recognized as a collectivist culture. In collectivist cultures there is a harmony between members of society (28). In these cultures, there are more frequent rules for social behavior than individualist cultures. In Asian countries, rigorous social rules apply to appropriate behavior in certain social situations (29). It is important for individuals in such communities to have an acceptable and positive evaluation of their social behavior (30). Thus it seems that social phobia in Iranians might be much higher than western counterparts. However, we believe this did not affect the study results since the items included in the BFNE-S are very straightforward and common among many different cultures.

This study had several limitations. For instance the sample size was relatively small and restricted to the students of a university and this limits the generalizability of the findings to the community, especially to the clinical samples. Further research is necessary to repeat the present findings and confirm the validity and reliability of the
BFNE-S in a clinical sample of Iranian patients with social phobia. In addition in current study, convergent validity was assessed by a generic questionnaire (the SPIN), while it is better to perform convergent validity by using a more specific measure of social phobia. Finally, one should note that for known groups comparison we compared male and female participants on the measure. This analysis has its own limitation since we could not really indicate that the BFNE items relate to the underlying construct similarly between male and female participants. In other words we are not sure that the items are demonstrating measurement invariance across sex, such that we know whether there are true differences or measurement artifacts.

\section{Conclusion}

The findings indicated that the Iranian version of the BFNE-S was a valid measure of fear of negative evaluation. Also the scale showed a unitary factor structure that confirms the theoretical basis for the BFNE. Indeed, applying the BFNE-S in future studies is recommended.

\section{Acknowledgments}

The authors would like to thanks Miss Neda Mahmoodi for help in data collection.

\section{Conflict of Interests}

The authors declare that they have no competing interests.

\section{References}

1. American Psychiatric Association. Diagnostic and statistical manual of mental disorders. (5th ed. Washington, DC; 2013.

2. Clark DM, Wells A. A cognitive model of social phobia. In Heimberg RG, Liebowitz MR, Hope DA, Schneier FR, editors. Social phobia: Diagnosis, assessment, and treatment. New York, NY: Guilford Press; 1995. 69-93.

3. Rapee RM, Heimberg RG. A cognitive-behavioral model of anxiety in social phobia. Behav Res Ther. 1997;35:741-756.

4. Weeks JW, Heimberg RG, Fresco DM, Hart TA, Turk CL, Schneier $\mathrm{FR}$, et al. Empirical validation and psychometric evaluation of the brief fear of negative evaluation scale in patients with social anxiety disorder. Psychol Assessment. 2005;17:179-190.

5. Watson D, Friend R. Measurement of social-evaluative anxiety. J Consult Clin Psych. 1969;33:448-457.

6. Leary MR. A brief version of the Fear of Negative Evaluation Scale. Pers Soc Psychol Bull. 1983;9:371-375.

7. Rodebaugh TL, Woods CM, Thissen DM, Heimberg RG, Chambless DL, Rapee RM. More information from fewer questions: the factor structure and item properties of the original and brief Fear of Negative Evaluation scale. Psychol Assess. 2004;16:169-181.

8. Carleton RN, Collimore KC, Asmundson GJG. Social anxiety and fear of negative evaluation: Construct validity of the BFNE-II. J Anxiety Disord. 2007;21:131-141.

9. Carleton RN, McCreary DR, Norton PJ, Asmundson GJG. Brief fear of negative evaluation scale-revised. Depress Anxiety. 2006;23:297303.

10. Collins KA, Westra H, Dozois DJA, Stewart SH. The validity of the brief version Of the Fear of Negative Evaluation Scale. J Anxiety Disord. 2005;19:345-359.

11. Tavoli A, Melyani M, Bakhtiari M, Ghaedi GH, Montazeri A. The Brief Fear of Negative Evaluation Scale (BFNE): translation and validation study of the Iranian version. BMC Psychiatry. 2009;9:42.

12. Duke D, Krishnan M, Faith M, Storch EA. The psychometric properties of the Brief. Fear of Negative Evaluation Scale. J Anxiety Disord. 2006;20:807-817.

13. Stopa L, Clark DM. Social phobia: comments on the viability and 
validity of an analogue research strategy and British norms for the fear of negative evaluation questionnaire. Behav Cogn Psychoth. 2001;29:423-430.

14. Carleton RN, Collimore KC, McCabe RE, Antony MM. Addressing revisions to the brief fear of negative evaluation scale: Measuring fear of negative evaluation across anxiety and mood disorders. J Anxiety Disord. 2011;25:822-828.

15. Connor KM, Davidson JRT, Curchill LE, Sherwood A, Foa E, Weisler RH. Psychometric properties of the Social Phobia Inventory. Br J Psychiat. 2000;176:379-386.

16. Rezaei Dogaheh E. Psychometric Properties of Farsi Version of the Social Phobia Inventory (SPIN). P Soc Behav Sci. 2013;84:763-768.

17. Costello AB, Osborne JW. Best practices in exploratory factor analysis: Four recommendations for getting the most from your analysis. Pract Assess Res Eval. 2005;10:1-9.

18. Wei J, Zhang C, Li Y, Xue S, Zhang J. Psychometric Properties of the Chinese Version of the Fear of Negative Evaluation Scale-Brief (BFNE) and the BFNE-Straightforward for Middle School Students. PloS One. 2015;10:e115948.

19. Gallego MJ. Brief version of the Fear of Negative Evaluation Scale Straightforward Items (BFNE-S): Psychometric properties in a Spanish population. Span J Psychol. 2010;13:981-989.

20. Ware JE, Brook RH, Davies-Avery, Williams KN, Stewart AL, Rogers WH, et al. Conceptualization and Measurement of Health for Adults in the Health Insurance Study: Vol. 1, Model of Health and Methodology. Santa Monica, CA: RAND Corporation; 1980.

21. Hooper D, Coughlan J, Mullen, M. Structural equation modelling: Guidelines for determining model fit. EJBRM. 2008;6:53-60.

22. Hajdúka M, Bolekováb V, Heretik A. Psychometric properties of Brief Fear of Negative Evaluation: Comparison of BFNE and BFNES. Annales Psychol. 2015;1:12-19.

23. Harpole JK, Levinson CA, Woods CM, Rodebaugh TL, Weeks JW, Brown PJ, et al. Assessing the straightforwardly-worded Brief Fear of Negative Evaluation Scale for differential item functioning across gender and ethnicity. J Psychopathol Behav. 2015;37:306-317.

24. Beidel DC, Turner SM, Morris TL. Social Phobia and Anxiety Inventory for Children (SPAI-C): User's manual. North Tonawanda, NY: Multi-Health Systems; 2000.

25. La Greca AM. Friends or foes? Peer influences on anxiety among children and adolescents. In: Silverman WK, Treffers PDA, editors. Anxiety Disorders in Children and Adolescents: Research, Assessment and Intervention. New York: Cambridge University Press; 2001. 159186.

26. Geravand F, Shokri O, Khodayi A, Amarayi M, Toolabi S. Standardization, validity and reliability of the brief fear of negative evaluation in adolescences in Tehran. J Psychol Stud. 2011;7:65-95. [In Persian]

27. Hofmann SG, Asnaani A. Cultural aspects in social anxiety and social anxiety disorder. Depress Anxiety. 2010;27:1117-1127.

28. Lucas RE, Diener E, Grob A. Cross-cultural evidence for the fundamental features of extraversion. J Pers Soc Psycho. 2000;79:452-468.

29. Argyle M. Rules for social relationships in four cultures. Aust J Psychol. 1986;38:309-318.

30. Suh E, Diener E, Oishi S, Triandis HC. The shifting basis of life satisfaction judgement across cultures: Emotions versus norms. J Pers Soc Psychol. 1998;74:482-493. 